\title{
Liver resection versus intensity-modulated radiation therapy for treatment of hepatocellular carcinoma with hepatic vein tumor thrombus: a propensity score matching analysis
}

\author{
Zhen-Hua Chen ${ }^{1 \#}$, Xiu-Ping Zhang ${ }^{1,2 \#}$, Shuang Feng ${ }^{3 \#}$, Jing-Kai Feng ${ }^{1}$, Zong-Tao Chai ${ }^{1}$, Wei-Xing Guo ${ }^{1}$, \\ Jie Shi ${ }^{1}$, Wan Yee Lau ${ }^{1,4}$, Yan Meng ${ }^{3}$, Shu-Qun Cheng ${ }^{1}$ \\ ${ }^{1}$ Department of Hepatic Surgery VI, Eastern Hepatobiliary Surgery Hospital, Second Military Medical University, Shanghai, China; ${ }^{2}$ Department of \\ Hepatobiliary and Pancreatic Surgical Oncology, The First Medical Center of Chinese People's Liberation Army (PLA) General Hospital, Beijing, \\ China; ${ }^{3}$ Department of Radiotherapy, Eastern Hepatobiliary Surgery Hospital, Second Military Medical University, Shanghai, China; ${ }^{4}$ Faculty of \\ Medicine, The Chinese University of Hong Kong, Hong Kong, China \\ Contributions: (I) Conception and design: SQ Cheng, WY Lau, Y Meng, ZH Chen, XP Zhang, S Feng; (II) Administrative support: SQ Cheng; (III) \\ Provision of study materials or patients: S Feng, WX Guo, J Shi; (IV) Collection and assembly of data: ZH Chen, XP Zhang, ZT Chai; (V) Data \\ analysis and interpretation: ZH Chen, XP Zhang, JK Feng; (VI) Manuscript writing: All authors; (VII) Final approval of manuscript: All authors. \\ \#These authors contributed equally to this work. \\ Correspondence to: Shu-Qun Cheng, MD, PhD. Department of Hepatic Surgery VI, Eastern Hepatobiliary Surgery Hospital, Second Military Medical \\ University, 225 Changhai Road, Shanghai 200433, China. Email: chengshuqun@aliyun.com.
}

Background: The presence of hepatic vein tumor thrombus (HVTT) is a major determinant of survival outcomes in hepatocellular carcinoma (HCC) patients. This study compared survival outcomes between liver resection (LR) and intensity-modulated radiation therapy (IMRT) in HCC patients with HVTT.

Methods: Data from patients who underwent LR or IMRT for HCC with HVTT at the Eastern Hepatobiliary Surgery Hospital were retrospectively analyzed. Their survival outcomes were compared before and after propensity score matching (PSM).

Results: Three hundred and seven HCC patients with HVTT who underwent either LR ( $\mathrm{n}=140)$ or IMRT $(\mathrm{n}=167)$ were enrolled. PSM matched 82 pairs of patients. The overall survival (OS) and recurrence-free survival (RFS) rates were significantly higher for patients in the LR group than those in the IMRT group. On subgroup analysis, significantly better survival outcomes were obtained after LR than IMRT in patients with peripheral type of HVTT (pHVTT) and major type of HVTT (mHVTT). However, similar survival outcomes were obtained after LR and IMRT when the HVTT had developed into inferior vena cava tumor thrombus (IVCTT).

Conclusions: LR resulted in significantly better survival outcomes in HCC patients with HVTT when compared to IMRT. Once the HVTT had developed IVCTT, LR and IMRT resulted in similarly bad survival outcomes.

Keywords: Hepatocellular carcinoma (HCC); hepatic vein tumor thrombus (HVTT); liver resection (LR); radiotherapy

Submitted Dec 12, 2019. Accepted for publication Mar 13, 2020.

doi: 10.21037/hbsn.2020.03.20

View this article at: https://dx.doi.org/10.21037/hbsn.2020.03.20

\section{Introduction}

Hepatocellular carcinoma (HCC) is the fifth most common cancer and the third leading cause of cancer-related death globally (1). HCC often invades macrovascular lumens such as the portal or hepatic venous branches to form a portal vein tumor thrombus (PVTT) or a 


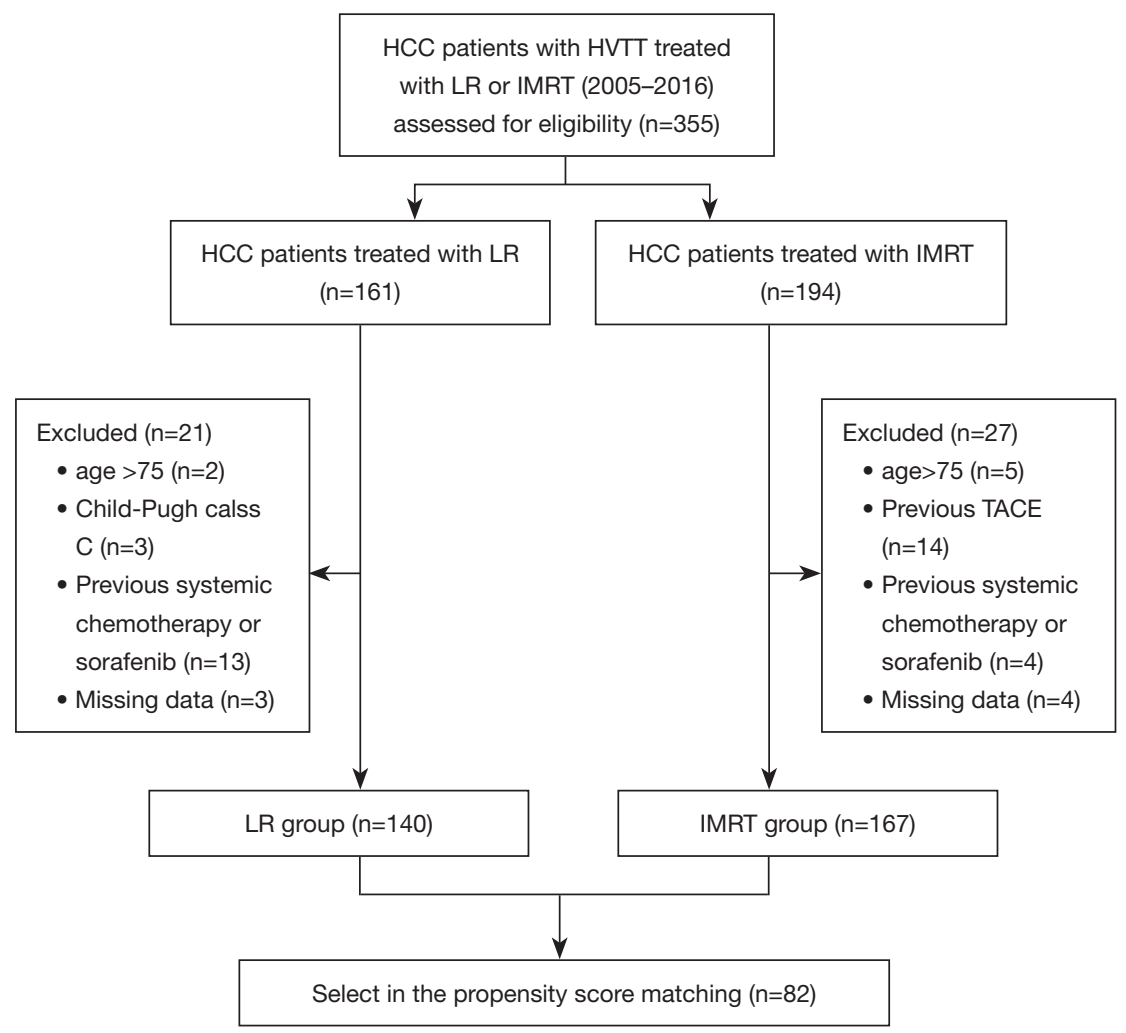

Figure 1 Flowchart of screening all HCC patients with HVTT for the study. HCC, hepatocellular carcinoma; HVTT, hepatic vein tumor thrombus; IMRT, intensity-modulated radiation therapy; LR, liver resection.

hepatic vein tumor thrombus (HVTT). The incidences of PVTT or HVTT have been reported to range in patients with HCC from $44 \%$ to $62.2 \%$ or from $1.4 \%$ to $4.9 \%$, respectively (2-4). Either PVTT or HVTT is a known significant risk factor affecting long-term survival for HCC patients $(3,5)$. Knowledge on HVTT is especially limited due to its low incidence. Invasion and extension of HVTT to the inferior vena cava (IVC) and right atrium (RA) can cause sudden death due to heart failure or massive pulmonary embolism (6). The best treatment strategy for HCC patients with HVTT remains controversial. The Barcelona Clinic for Liver Cancer (BCLC) Staging System and Treatment Guidelines consider presence of HVTT or PVTT to be at an advanced stage of HCC, with almost zero hope for a cure. Sorafenib is recommended as the only treatment option $(7,8)$. A phase III randomized controlled trial reported a median survival time (MST) of only 6.5 months for these patients (7). Furthermore, recent studies from Asia showed that liver resection (LR) can result in better survival outcomes compared to nonsurgical treatment in selected HCC patients with HVTT (3,9-11).

Improvements in radiation therapy (RT) which include image-guided radiation therapy (IGRT) and intensitymodulated radiation therapy (IMRT) have allowed increasing use of external RT in treatment of HCC. Retrospective and prospective studies have showed RT to be safe and effective in patients with advanced and unresectable HCC (12-14) or with macroscopic portal/ hepatic venous invasion (15-17). Furthermore, RT can be given as an outpatient basis, and it seldom produces grade 3 or higher liver, gastrointestinal, or hematological toxicities $(18,19)$.

In this large-scale retrospective study, the long-term survival outcomes of HCC patients with HVTT who underwent LR or IMRT as their primary treatments were analyzed. To minimize potential biases which are inherent to retrospective studies, propensity score matching (PSM) analysis was used to determine the differences in longterm survival outcomes between these two treatments. 
We present the following article in accordance with the STROBE reporting checklist (available at https://hbsn. amegroups.com/article/view/10.21037/hbsn.2020.03.20/rc).

\section{Methods}

\section{Patients enrollment}

As shown in Figure 1, 355 consecutive HCC patients with HVTT who underwent LR or IMRT as primary treatments at the Eastern Hepatobiliary Surgery Hospital from January 2005 to December 2016 were included in this study. Fortyeight patients were excluded from the study because they met the exclusion criteria. Thus, 307 patients (LR group, $\mathrm{n}=140$; IMRT group, $\mathrm{n}=167$ ) formed the basis of this study. After PSM with a 1:1 ratio matching, there were 82 patients in each of the LR and the IMRT groups. The demographic, clinical and pathological data, and survival outcomes were recorded prospectively in a HCC database and analyzed retrospectively. The study was conducted in accordance with the Declaration of Helsinki (as revised in 2013). The study was approved by the Research Ethics Committee of the Eastern Hepatobiliary Surgery Hospital (Permit Number: HBHKY-2019-001-017). Informed consent was obtained from all the patients prior to treatment.

The extent of HVTT was categorized as: a tumor thrombous in a peripheral hepatic vein precluding the microscopic HVTT (pHVTT), in a major hepatic vein (mHVTT), or in the inferior vena cava (IVCTT) (3). In HCC patients who had HVTT coexisting with PVTT, the extent of PVTT was classified according to the Cheng's classification of the extent of PVTT in the portal vein (20): Type I, tumor thrombus in the segmental branches of the portal venous system or above; Type II, tumor thrombus extending to the right or the left portal vein; Type III, tumor thrombus extending to the main portal vein; and Type IV, tumor thrombus extending to the main portal vein and the superior mesenteric vein.

\section{Inclusion and exclusion criteria}

The presence of HVTT and PVTT was identified by preoperative Doppler ultrasonography, enhanced computed tomography (CT) or magnetic resonance image (MRI), and was later confirmed by final histopathological examination in the group of patients who underwent LR.

The inclusion criteria for surgery group were: (I) age between 18 and 75 years; (II) HCC patients who underwent
LR as the primary treatment; (III) liver function of ChildPugh class A or selected B (score $\leq 7$ ); (IV) MELD score $<11$; and (V) resectable HCC which was defined as HCC which the potential to be completely removed of all macroscopic tumor tissues and with a future liver remnant of a volume sufficient to sustain life after surgery as assessed by our surgical team (21). The MELD score was $7.9 \pm 2.1$ for the surgery group, and $8.4 \pm 2.5$ for the IMRT group. There were $53(37.9 \%)$ patients underwent TACE and 13 (9.3\%) patients underwent sorafenib along with LR $(n=140)$.

The exclusion criteria were: (I) Liver function of ChildPugh class C; (II) underwent preoperative anti-cancer treatment which included sorafenib, radiofrequency ablation, TACE, and/or ethanol injection; (III) presence of distant metastases; and (IV) incomplete data.

\section{$L R$}

LR was performed as previously reported by us (22). Only patients with liver functions of Child-Pugh A or selected B were offered surgery. After exploration, routine intraoperative ultrasonography was carried out to determine the location and extent of HVTT, IVCTT, PVTT, and to rule out any preoperatively undetected tumors in the future liver remnant. The abdominal cavity was carefully searched for the extent of local disease, extrahepatic metastases, and peritoneal seeding. For patients with a tumor thrombus in a portal vein, the blood inflow of the liver was occluded using the Pringle's maneuver at a site distal to the thrombus. The clamp crushing method was used to carry out liver transection. Anatomical LR with en bloc thrombectomy was our preferred surgical method. As an alternative, non-anatomical resection was used in patients when en bloc resection was not technically feasible. For PVTT, thrombectomy was performed according to the types of PVTT. For patients with Type I or II PVTT, the PVTT was resected en bloc with the specimen (22). After flushing with normal saline and confirmation with intraoperative ultrasonography that no tumor thrombus remained, the vascular incision was closed with a continuous suture. For HVTT, the tumor thrombus was either resected en bloc with the LR or it was extracted out of the vascular lumen depending on its location and extent. For HVTT with a tumor thrombus extending to the IVC, the infrahepatic and suprahepatic IVC were exposed and encircled with umbilical tapes for total hepatic vascular exclusion (THVE). Before the initiation of THVE, test clamping of the IVC was repeated several times to determine whether a 
venovenous bypass was necessary. During THVE, the patient's hemodynamic condition was carefully monitored and aggressively treated by the anesthesiology team. In patients with an IVC tumor thrombus extending above the diaphragm, the supradiaphragmatic IVC was dissected by blunt and sharp dissections through a vertical incision in the diaphragm (23). The supradiaphragmatic IVC was then encircled and controlled by a tape. When an IVC tumor thrombus had extended into the RA, a median sternotomy was made, and cannulations of the ascending aorta, superior vena cava, and right femoral vein were carried out to perform extracorporeal circulation (ECC).

\section{IMRT}

IMRT was performed in patients who were not eligible to undergo LR because of inoperable tumors. Some patients preferred IMRT because of the surgery refusal. The inclusion criteria were: (I) age between 18 and 75 years; (II) HCC patients who underwent IMRT as the primary treatment; (III) liver function of Child-Pugh class A or selected B (score $\leq 7$ ); (IV) absence distant metastases. The MELD score was $8.4 \pm 2.5$ for the IMRT group. There were $65(38.9 \%)$ patients underwent TACE, 18 (10.8\%) patients underwent sorafenib along with IMRT ( $\mathrm{n}=167)$. A computed tomographic scan was performed with the patient in a supine position, with thermoplastic mask immobilization to reduce setup uncertainty and to restrain liver motion caused by abdominal breathing. Preoperative MRI scans were used to optimize target and normal structure delineation. The clinical target volume (CTV) included the primary tumor and tumor thrombus. Considering respiratory liver motion and setup errors in four-dimensional (4D) CT, the planning target volume (PTV) was defined by expanding the CTV by $0.5 \mathrm{~cm}$ in the anterior-posterior and left-right directions and by $1.0 \mathrm{~cm}$ in the cranial-caudal direction. IMRT plans were then generated for each patient. In all patients, the goal was for at least $95 \%$ of the clinical treatment volume to receive $100 \%$ of the dose. The plans were optimized independently and reviewed by at least a dosimetrist and a physicist. The prescribed doses to the initial PTV ranged from 50 to $67 \mathrm{~Gy}$ (median $58 \mathrm{~Gy}$ ), given in daily doses of 2.0-2.2 Gy. The biologically effective dose (BED) ranged from 61.0 to $82.5 \mathrm{~Gy}$ (median $68.2 \mathrm{~Gy}, \alpha / \beta=10$ ). The dose was selected so as not to cause too much damage to the vascular components near the HVTT, which would result in postoperative complications

\section{Follow-up}

Patients were followed-up once every 3 to 4 months until death or dropout from the follow-up program. Follow-up examinations were conducted using laboratory tests (serum AFP, liver function and complete blood count), abdominal ultrasonography and contrast-enhanced CT. A diagnosis of HCC recurrence was based on CT and/or magnetic resonance imaging with or without a raised serum $\alpha$-fetoprotein (AFP) level. Patients with recurrence or metastasis were treated by TACE, radiofrequency ablation therapy, hepatectomy, systemic chemotherapy, or sorafenib therapy. Therapy was decided based on hepatic function, performance status, and economic conditions. Overall survival (OS) was defined as the interval between operation/IMRT and death due to any cause or the last follow-up. Recurrence-free survival (RFS) was defined as the interval from the date between operation/IMRT to the date when HCC recurrence was diagnosed. OS and RFS were the primary outcomes of this study. This study was censored on May 1, 2018.

\section{Statistical analysis}

For comparisons between baseline variables, the Wilcoxon rank-sum test was used for continuous variables and the chi-squared test for categorical variables. Survival curves were conducted using the Kaplan-Meier method, and the differences were analyzed using the log-rank test. Prognostic factors found to be significant on univariate analysis $(\mathrm{P}<0.05)$ were subjected to multivariate analysis using the Cox proportional hazards regression model. The PSM method was used to control selection bias and to create demographically and clinically comparable cohorts. The propensity score was estimated using the non-parsimonious multivariate logistic regression according to age, sex, ChildPugh class, hepatitis B virus infection, satellite nodules, AFP, CEA, CA-19.9, tumor diameter, tumor number, extent of PVTT, and extent of HVTT. Patients were matched at a 1:1 ratio using the caliper matching method within 0.2 of the standard deviation of the logit of the PS. Statistical analyses were performed using the $\mathrm{R}$ statistical package, Version 3.4.3 (R Development Team, Vienna, Austria).

\section{Results}

\section{Patient characteristics}

The characteristics of the patients in the LR and IMRT 
Table 1 The clinicopathological features of all patients in our study before PSM ( $\mathrm{n}=307)$

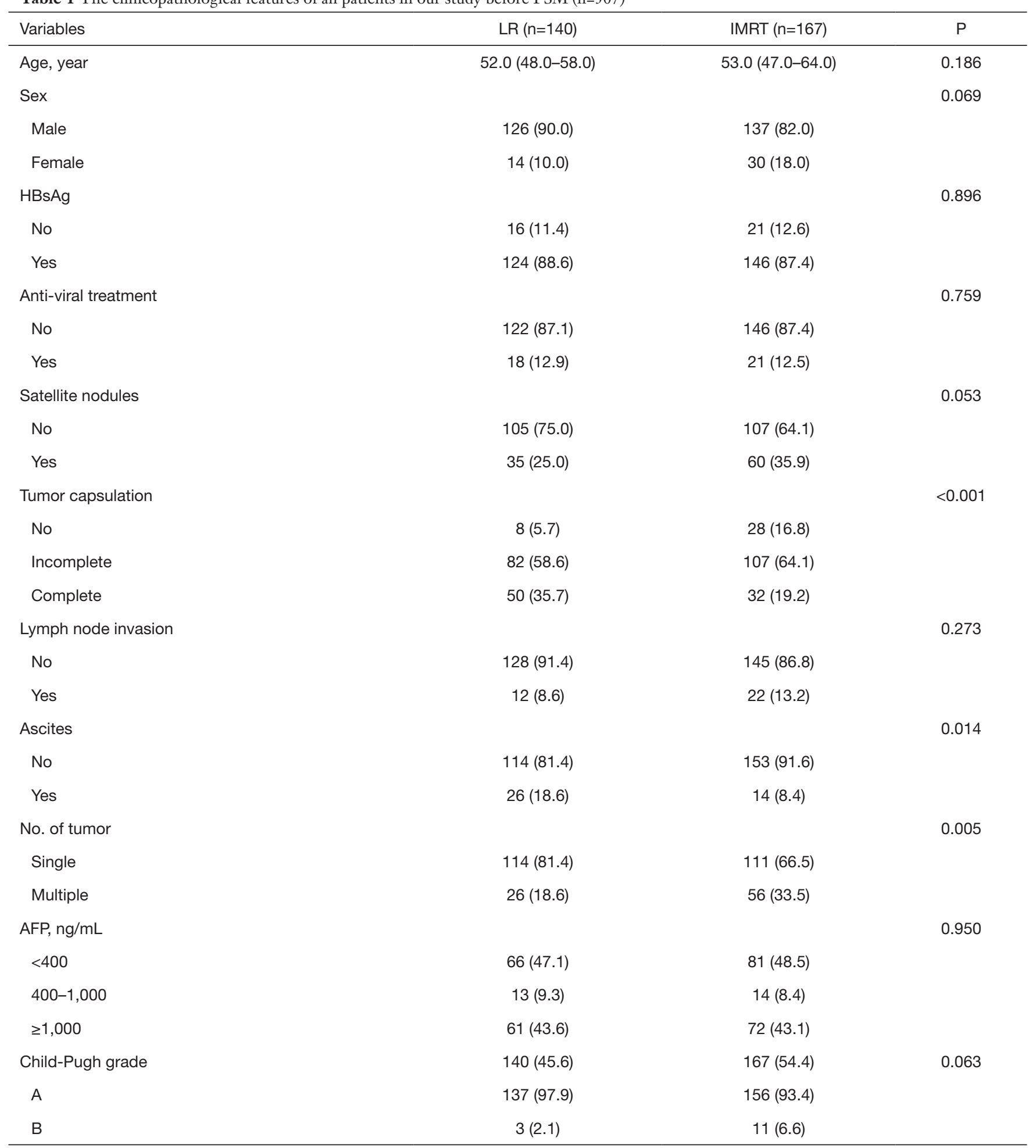

Table 1 (continued) 
Table 1 (continued)

\begin{tabular}{|c|c|c|c|}
\hline Variables & $\operatorname{LR}(n=140)$ & IMRT $(n=167)$ & $P$ \\
\hline PT, s & $12.5(11.6-13.4)$ & $12.4(11.7-12.9)$ & 0.141 \\
\hline $\mathrm{ALT}, \mathrm{U} / \mathrm{L}$ & $37.7(25.0-63.5)$ & $40.0(24.6-70.0)$ & 0.460 \\
\hline TP, g/L & $67.75(62.4-71.8)$ & $67.7(63.9-71.6)$ & 0.844 \\
\hline AST, U/L & $44.0(31.0-65.0)$ & $52.0(36.0-81.0)$ & 0.005 \\
\hline GGT, U/L & $117.5(53.5-203.0)$ & $140.0(75.0-237.0)$ & 0.046 \\
\hline ALP, U/L & $107.0(85.0-143.0)$ & $104.0(88.0-152.0)$ & 0.748 \\
\hline PLT, $10^{9} / \mathrm{L}$ & $159.0(86.0-203.0)$ & $158.0(109.0-205.0)$ & 0.665 \\
\hline Extent of HVTT & & & 0.319 \\
\hline pHVTT & $42(30.0)$ & $54(32.3)$ & \\
\hline mHVTT & $54(38.6)$ & $51(30.5)$ & \\
\hline IVCTT & $44(31.4)$ & $62(37.1)$ & \\
\hline Extent of PVTT & & & 0.021 \\
\hline No & $89(63.6)$ & $80(47.9)$ & \\
\hline Type I & $30(21.4)$ & $48(28.7)$ & \\
\hline Type II & $21(15.0)$ & $39(23.4)$ & \\
\hline
\end{tabular}

Data are the median (IQR) or number (percentage) unless otherwise indicated. PSM, propensity score matching; IMRT, intensitymodulated radiation therapy; LR, liver resection; HBsAg, hepatitis B surface antigen; AFP, $\alpha$-fetoprotein; PT, prothrombin time; ALT, alanine aminotransferase; TP, total protein; ALB, albumin; AST, aspartate aminotransferase; GGT, $\gamma$-glutamyltransferase; ALP, alkaline phosphatase; PLT, platelet; TBIL, total bilirubin; DBIL, direct bilirubin; HVTT, hepatic vein tumor thrombus; pHVTT, peripheral type of HVTT; mHVTT, major type of HVTT; IVCTT, inferior vena cava tumor thrombus; PVTT, portal vein tumor thrombus.

groups before PSM are shown in Table 1. Patients in the IMRT group had higher rates of absence of ascites, multiple tumors, large tumor diameter, high levels of AST and GGT, and presence of PVTT than the patients in the LR group (all $\mathrm{P}<0.05)$. After PS matching, all these clinicopathological features became well-balanced between the two groups (Table 2).

\section{Univariate and multivariate analyses of predictors of $O S$ and RFS}

Before PSM, uni- and multi-variate analyses demonstrated that presence of ascites $(\mathrm{P}=0.029)$, tumor diameter $(\mathrm{P}=0.005)$, extent of HVTT $(\mathrm{P}<0.001)$, extent of PVTT $(\mathrm{P}=0.001)$, and treatment modality $(\mathrm{P}<0.001)$ were independently related to OS (Table 3), whereas tumor diameter $(\mathrm{P}=0.021)$, level of AST $(\mathrm{P}=0.026)$, extent of HVTT $(\mathrm{P}<0.001)$, extent of PVTT $(\mathrm{P}=0.001)$, and treatment modality $(\mathrm{P}<0.001)$ were independently related to RFS (Table S1).

After PSM, uni- and multi-variate analyses demonstrated that extent of HVTT $(\mathrm{P}<0.001)$, and treatment modality $(\mathrm{P}<0.001)$ were independently related to OS (Table $\mathrm{S} 2$ ), whereas presence of ascites $(\mathrm{P}=0.029)$, extent of HVTT $(\mathrm{P}<0.001)$, and treatment modality $(\mathrm{P}<0.001)$ were independently related to RFS (Table S3).

\section{Survival analysis before and after PSM}

Before PSM, the median OS times (MOST 95\% CI) 
Table 2 The clinicopathological features of all patients in our study after PSM ( $\mathrm{n}=164)$

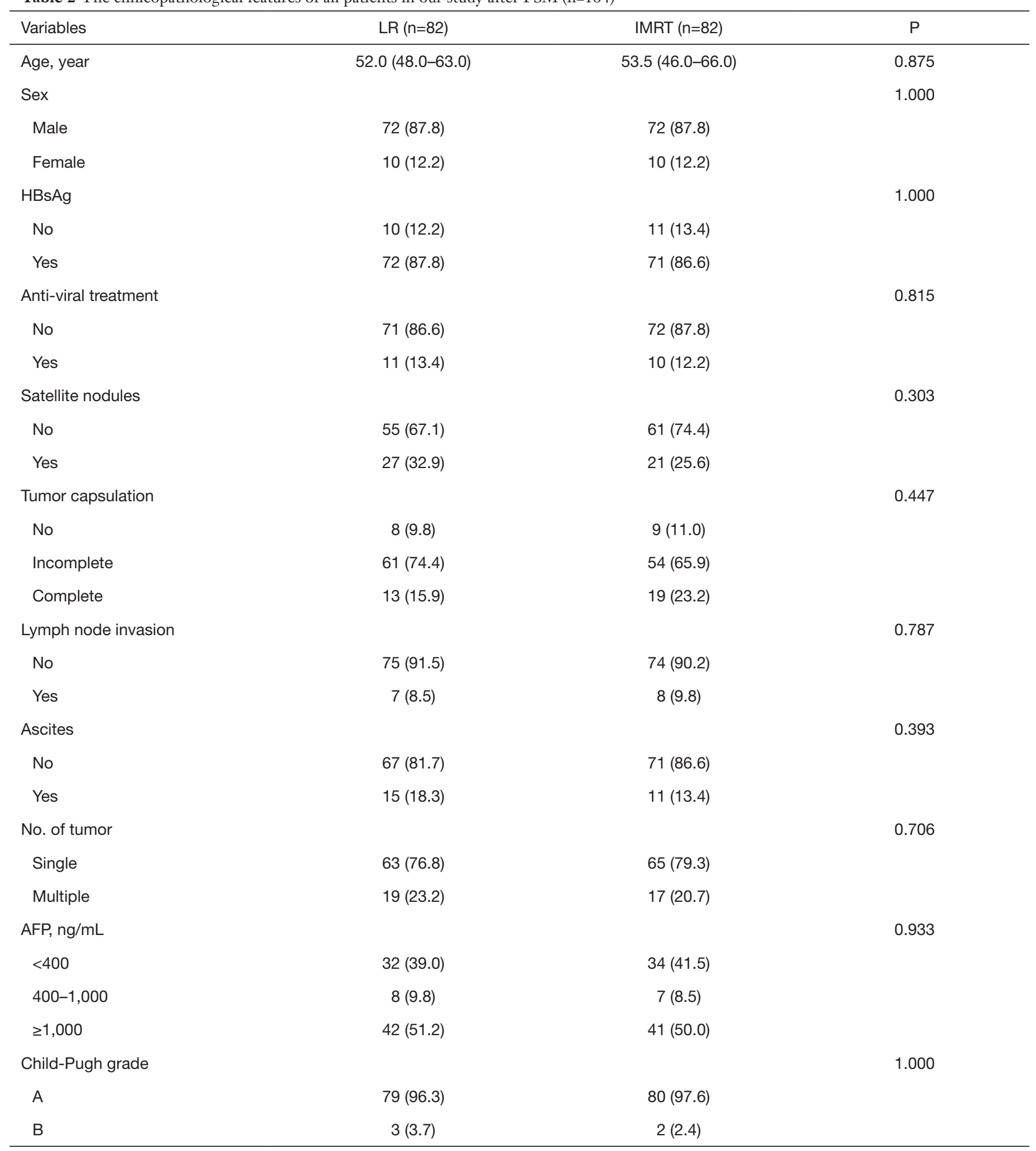

Table 2 (continued) 
Table 2 (continued)

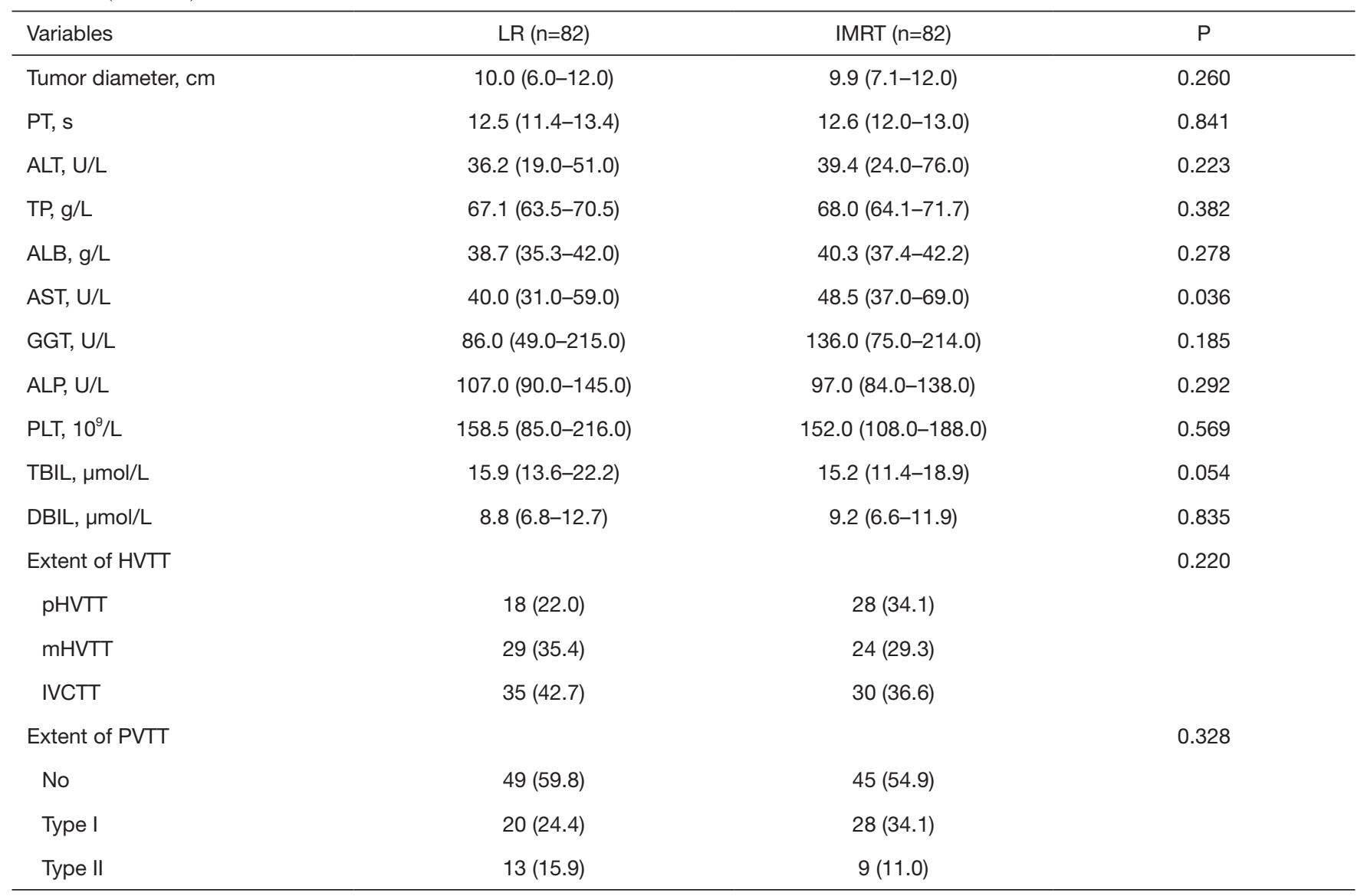

Data are the median (IQR) or number (percentage) unless otherwise indicated. PSM, propensity score matching; IMRT, intensitymodulated radiation therapy; LR, liver resection; HBsAg, hepatitis B surface antigen; AFP, $\alpha$-fetoprotein; PT, prothrombin time; ALT, alanine aminotransferase; TP, total protein; ALB, albumin; AST, aspartate aminotransferase; GGT, $\gamma$-glutamyltransferase; ALP, alkaline phosphatase; PLT, platelet; TBIL, total bilirubin; DBIL, direct bilirubin; HVTT, hepatic vein tumor thrombus; pHVTT, peripheral type of HVTT; mHVTT, major type of HVTT; IVCTT, inferior vena cava tumor thrombus; PVTT, portal vein tumor thrombus.

of the LR and IMRT groups were $21.0(17.0-25.9)$ and 16.5 (14.6-18.5) months, respectively. The LR group had significantly higher OS rates than the IMRT group (1-year, $67.5 \%$ vs. $66.8 \%$; 2 -year, $45.0 \%$ vs. $29.9 \%$; 3 -year, $20.7 \%$ vs. $10.6 \%$; 5 -year, $7.8 \%$ vs. $1.0 \% ; \mathrm{P}=0.004$, Figure $2 A$ ). The median RFS times (MRFST 95\% CI) of the LR and IMRT groups were $13.0(10.0-15.5)$ and $9.7(6.6-12.5)$ months, respectively. The LR group had significantly better RFS rates than the IMRT group (1-year, $51.8 \%$ vs. $43.7 \%$; 2 -year, $21.5 \%$ vs. $13.9 \%$; 3 -year, $7.5 \%$ vs. $1.4 \%$; 5 -year, $2.5 \%$ vs. $0 \% ; \mathrm{P}=0.004$, Figure $2 B$ ).

After PSM, the MOST (95\% CI) of the two groups were $22.0(17.5-25.9)$ and $16.8(14.0-21.0)$ months, respectively. The OS rates were significantly better in the LR group than the IMRT group (1-year, $70.4 \%$ vs. $67.7 \%$; 2-year,
44.5\% vs. $29.4 \%$; 3 -year, $17.6 \%$ vs. $9.4 \%$; 5 -year, $6.5 \%$ vs. $0 \% ; \mathrm{P}=0.018$, Figure 2C). The MRFST (95\% CI) of the two groups were 14.5 (10.0-17.0) and 10.0 (6.3-13.4) months, respectively. The RFS rates were significantly better in the LR group than the IMRT group (1-year, 55.4\% vs. 45.8\%; 2 -year, $21.4 \%$ vs. $14.0 \%$; 3 -year, $6.1 \%$ vs. $1.4 \%$; 5 -year, $1.5 \%$ vs. $0 \% ; \mathrm{P}=0.034$, Figure $2 D)$.

\section{Subgroup analysis on the survival of patients with HVTT (pHVTT and $m H V T T$ ), IVCTT, and PVTT}

For HCC patients with HVTT (pHVTT and mHVTT), the OS rates were significantly higher in the LR group than the IMRT group before PSM (1-year, $79.2 \%$ vs. $63.8 \%$; 2 -year, $55.2 \%$ vs. $35.2 \%$; 3 -year, $23.7 \%$ vs. $14.6 \%$; 
Table 3 Univariate and multivariable analysis for OS in HCC patients with HVTT before PSM (n=307)

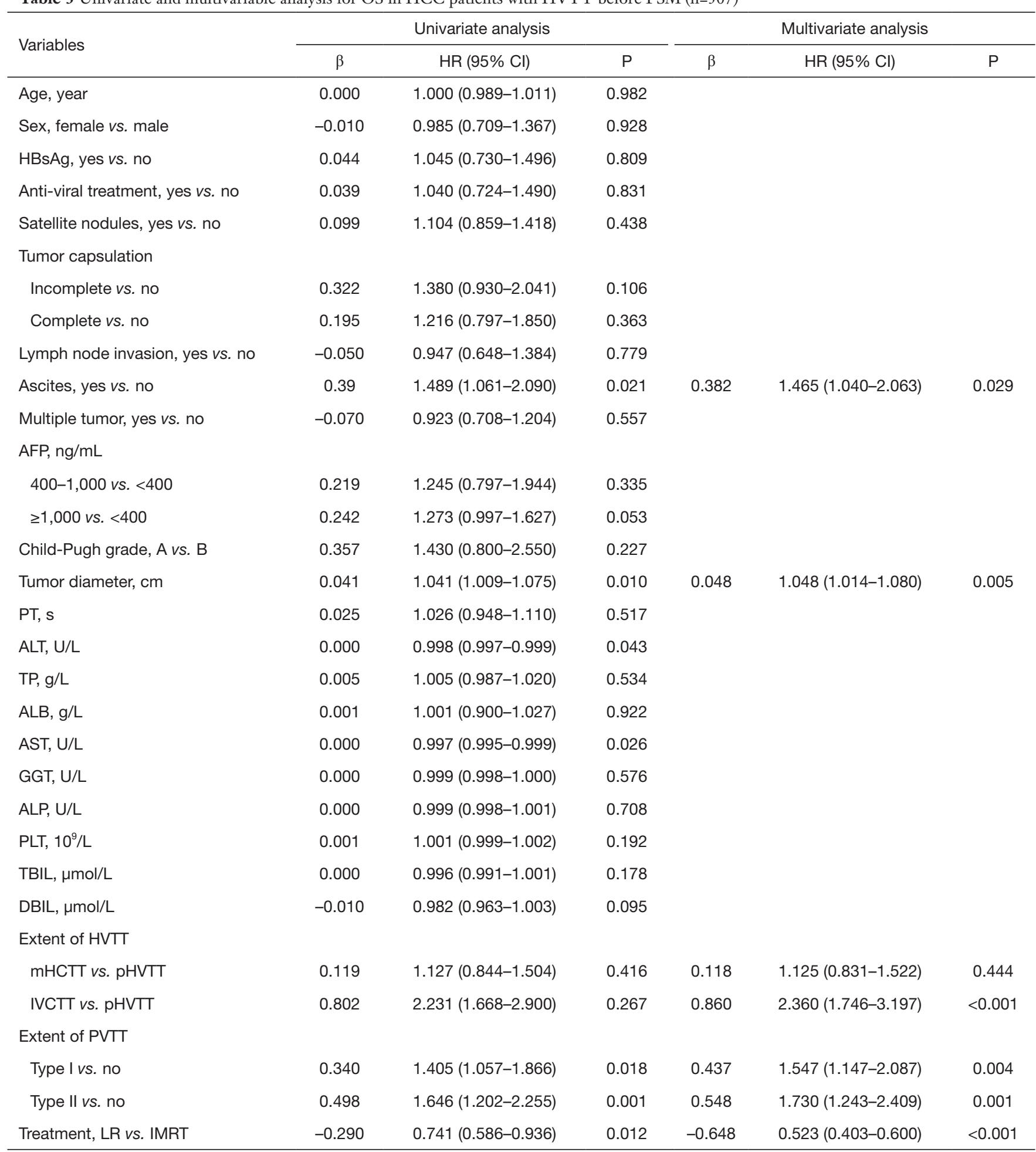

OS, overall survival; HCC, hepatocellular carcinoma; HVTT, hepatic vein tumor thrombus; PSM, propensity score matching; IMRT, intensity-modulated radiation therapy; LR, liver resection; HBsAg, hepatitis B surface antigen; AFP, $\alpha$-fetoprotein; PT, prothrombin time; ALT, alanine aminotransferase; TP, total protein; ALB, albumin; AST, aspartate aminotransferase; GGT, $\gamma$-glutamyltransferase; ALP, alkaline phosphatase; PLT, platelet; TBIL, total bilirubin; DBIL, direct bilirubin; pHVTT, peripheral type of HVTT; mHVTT, major type of HVTT; IVCTT, inferior vena cava tumor thrombus; PVTT, portal vein tumor thrombus. 
A

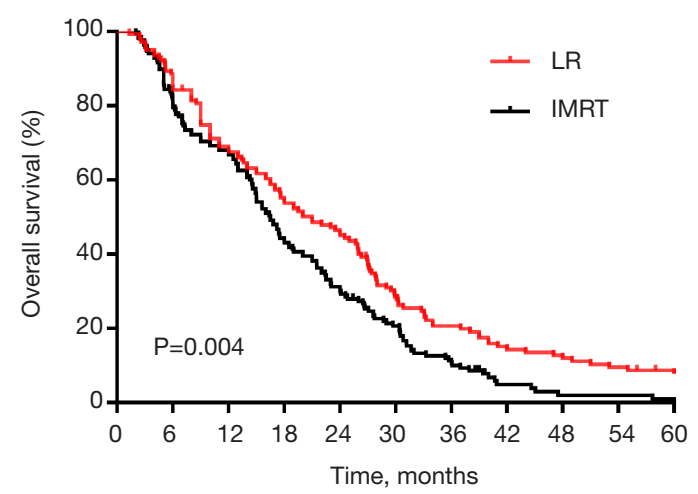

$\begin{array}{lccccccccccc}\text { LR } & 140 & 123 & 95 & 76 & 64 & 37 & 26 & 19 & 16 & 12 & 9 \\ \text { IMRT } & 167 & 135 & 112 & 73 & 48 & 31 & 17 & 5 & 2 & 2 & 1\end{array}$

C

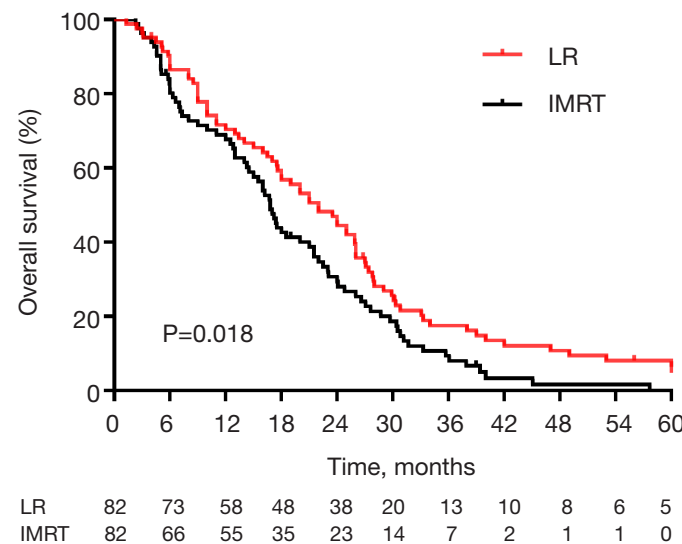

B
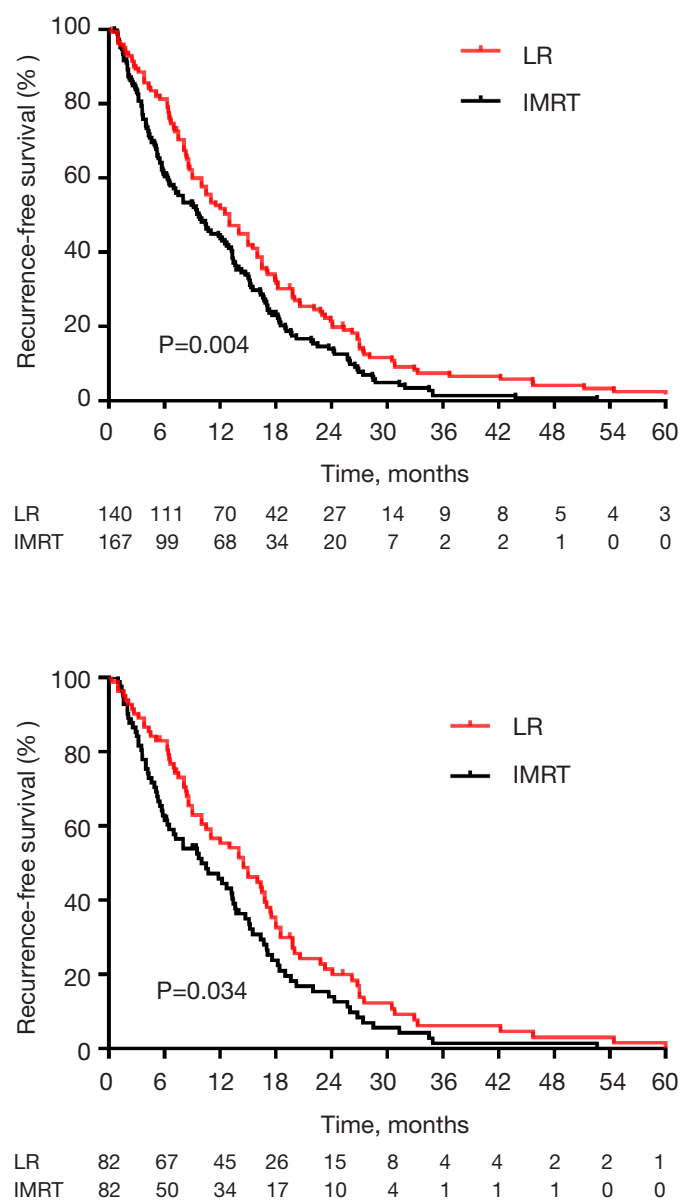

Figure 2 Kaplan-Meier analysis of OS and RFS in all HCC patients with HVTT. (A) The OS for HCC patients after LR or IMRT (140 vs. 167 patients) before PSM ( $\mathrm{P}=0.004)$; (B) the RFS for HCC patients after LR or IMRT (140 vs. 167 patients) before PSM (P=0.004); (C) the OS for HCC patients after LR or IMRT (82 vs. 82 patients) after PSM ( $\mathrm{P}=0.018)$; (D) the RFS for HCC patients after LR or IMRT (82 vs. 82 patients) after PSM ( $\mathrm{P}=0.034)$. OS, overall survival; RFS, recurrence-free survival; HCC, hepatocellular carcinoma; HVTT, hepatic vein tumor thrombus; IMRT, intensity-modulated radiation therapy; LR, liver resection; PSM, propensity score matching.

5 -year, $7.1 \%$ vs. $0 \% ; \mathrm{P}=0.002$, Figure $3 A$ ). The RFS rates in the LR group were significantly higher than the IMRT group before PSM (1-year, 62.2\% vs. 45.4\%; 2-year, $28.9 \%$ vs. $16.5 \%$; 3 -year, $10.5 \%$ vs. $2.5 \%$; 5 -year, $3.5 \%$ vs. $0 \%$; $\mathrm{P}=0.003$, Figure $3 B$ ). After PSM, the OS and RFS rates were also significantly higher in the LR group than the IMRT group (OS: 1-year, $82.2 \%$ vs. 62.2\%; 2-year, 55.6\% vs. $28.9 \%$; 3 -year, $24.9 \%$ vs. $11.0 \%$; 5 -year, $3.3 \%$ vs. $0 \%$; $\mathrm{P}=0.014$, Figure $3 C$; RFS: 1-year, $64.4 \%$ vs. $46.4 \%$; 2-year, $28.7 \%$ vs. $15.3 \%$; 3 -year, $10.5 \%$ vs. $3.1 \%$; 5 -year, $2.6 \%$ vs. $0 \% ; \mathrm{P}=0.037$, Figure 3D). For HCC patients with pHVTT and mHVTT, LR had better OS and RFS rates than IMRT as shown in Figure $\mathrm{S} 1(\mathrm{P}=0.018$ and $\mathrm{P}=0.027)$.

For HCC patients with IVCTT, there were no significant differences in the OS and RFS rates between the two groups of patients before PSM (OS: $\mathrm{P}=0.986$, Figure $4 A$; RFS: $\mathrm{P}=0.557$, Figure $4 B$ ). After PSM, there were also no significant difference in OS and RFS rates for the two groups of patients (OS: $\mathrm{P}=0.821$, Figure $4 C$; RFS: $\mathrm{P}=0.657$, Figure $4 D$ ).

For patients who underwent LR, the median OS and RFS were significantly higher in patients without PVTT than those with PVTT $(\mathrm{P}<0.001$, Figure S2). Similarly, for patients who underwent IMRT, the no-PVTT subgroup had higher median OS and RFS than the PVTT group $(\mathrm{P}<0.001$, Figure $\mathrm{S} 2)$. 
A

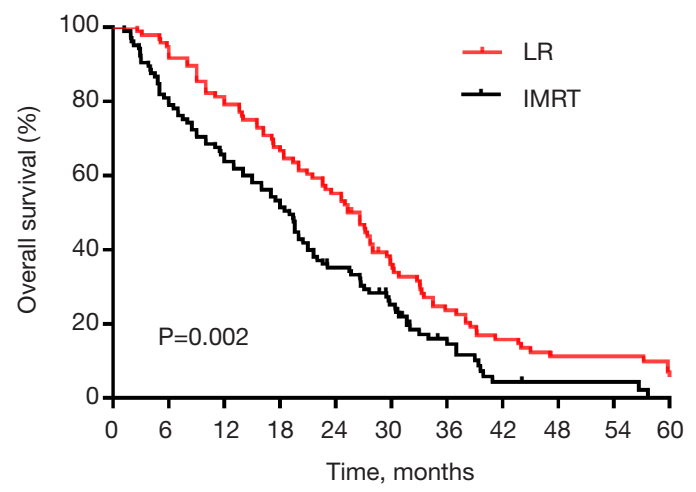

B

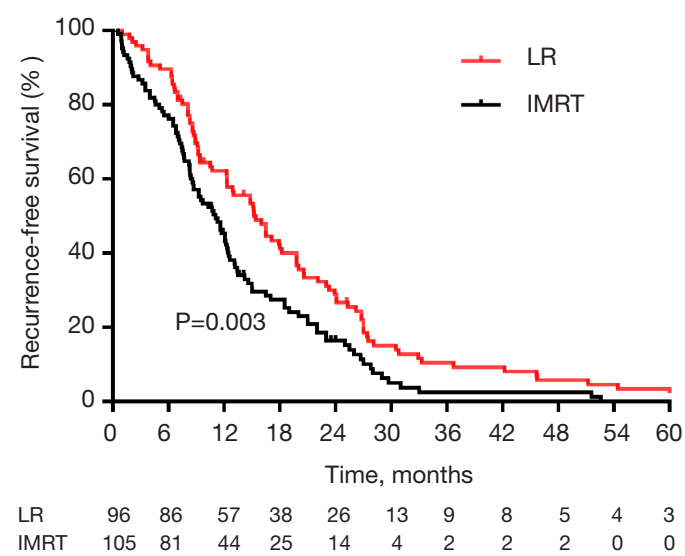

C

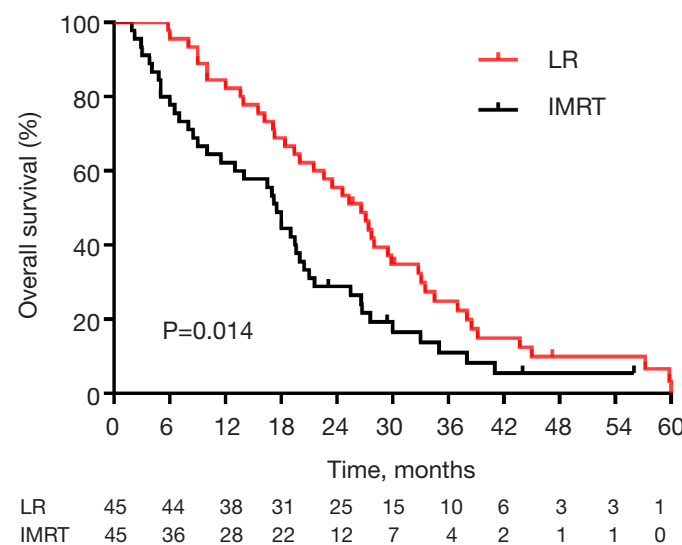

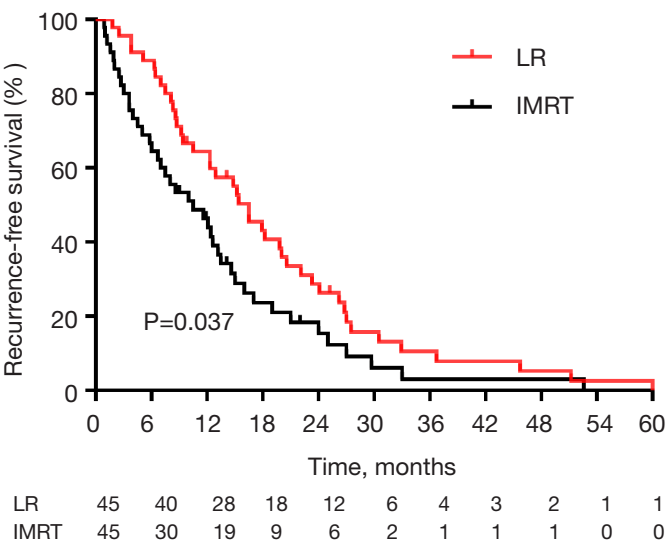

Figure 3 Kaplan-Meier subgroup analysis of OS and RFS in HCC patients with HVTT (pHVTT and mHVTT). (A) The OS for HCC patients after LR or IMRT (96 vs. 105 patients) before PSM ( $\mathrm{P}=0.002)$; (B) the RFS for HCC patients after LR or IMRT (96 vs. 105 patients) before PSM ( $\mathrm{P}=0.003)$; (C) the OS for HCC patients after LR or IMRT (45 vs. 45 patients) after PSM ( $\mathrm{P}=0.014)$; (D) the RFS for HCC patients after LR or IMRT (45 vs. 45 patients) after PSM ( $\mathrm{P}=0.037)$. OS, overall survival; RFS, recurrence-free survival; HCC, hepatocellular carcinoma; HVTT, hepatic vein tumor thrombus; pHVTT, peripheral type of HVTT; mHVTT, major type of HVTT; IMRT, intensity-modulated radiation therapy; LR, liver resection; PSM, propensity score matching.

\section{Adverse events of IMRT}

Table S4 summarizes the toxicities of the CTCAE Grade 3-5 for all the patients who underwent IMRT. Fatigue, anorexia, and nausea were the most common acute toxicities, but most of them were in the CTCAE Grade 1 or 2. For gastrointestinal complications, 6 patients (3.6\%) developed CTCAE Grade 3 mucositis or ulcers within the radiation field. No treatment-related deaths or serious adverse events were observed in this study.

\section{Detailed procedures and outcomes of $L R$}

As shown in Table S5, there were 115 (82.1\%) patients undergoing R0 resection, 89 (63.6\%) patients major hepatectomy, and $86(61.4 \%)$ anatomical resection. The median hospital stay was 19 [14-24] days. Morbidity and mortality were described according to the ClavienDindo classification. The majority of complications of LR belonged to Clavien-Dindo grade I (15.7\%) or grade II (12.9\%). A total of seven patients (5.0\%) experienced grade III complications due to pulmonary infection $(\mathrm{n}=2)$ and pulmonary embolism $(\mathrm{n}=5)$. Two patients $(1.4 \%)$ experienced severe liver failure and received comprehensive treatment in intensive. No one died from complications of LR during the study period. There were only 5 patients experienced pulmonary embolism in the surgery group. 
A

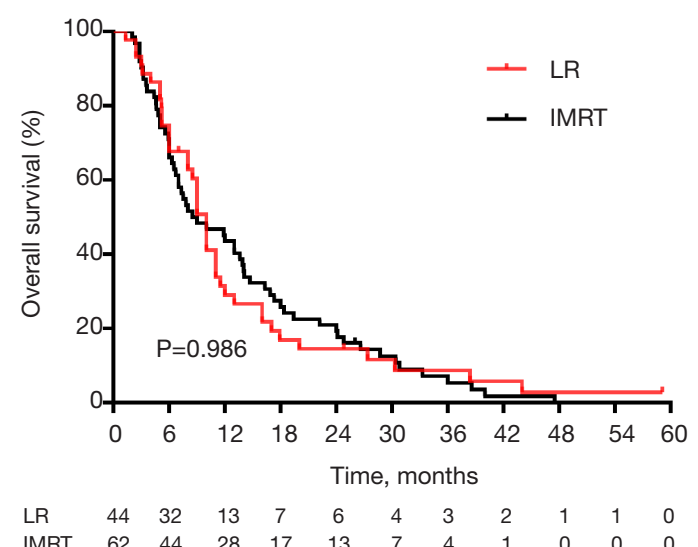

C

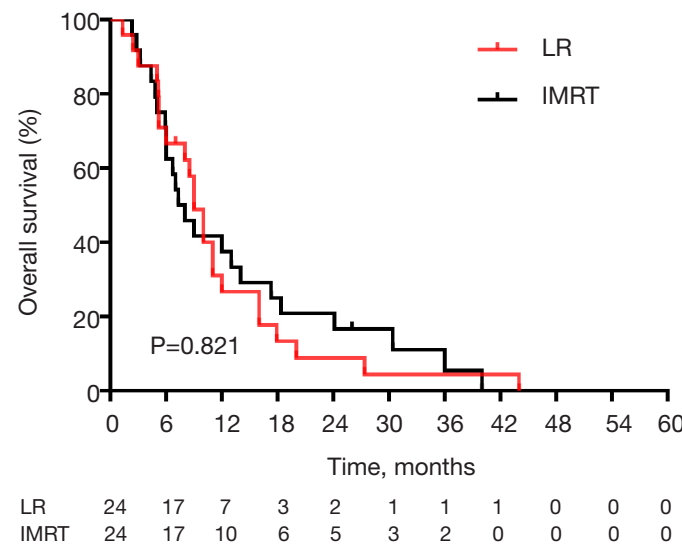

B
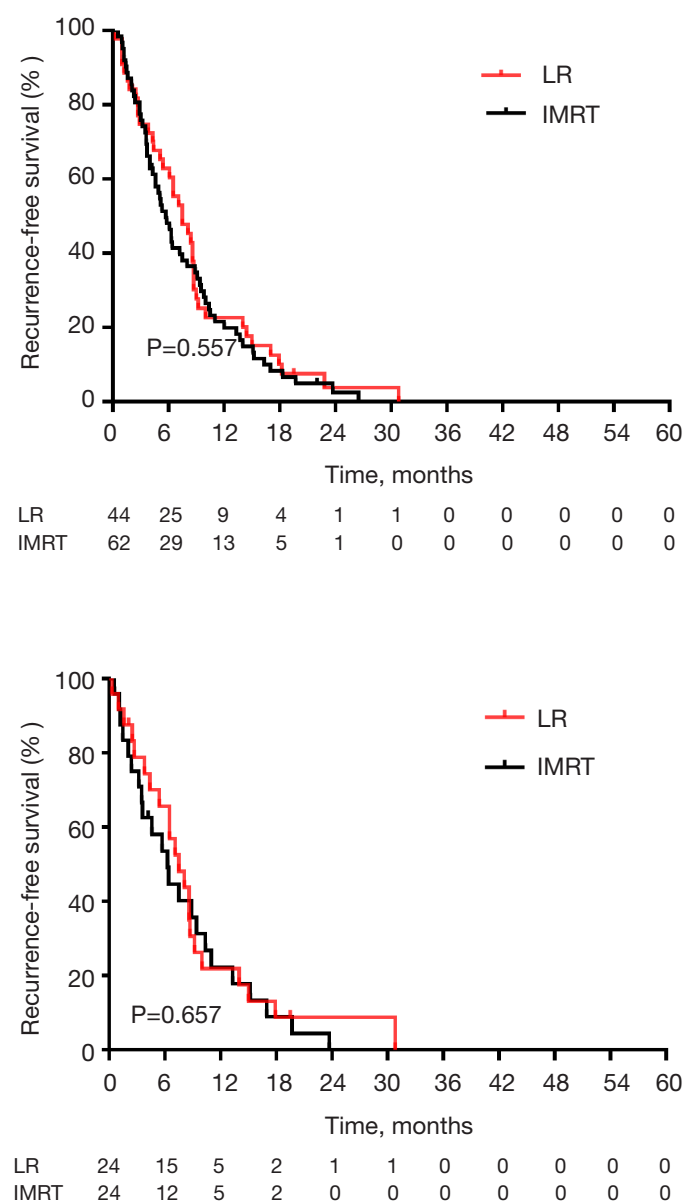

Figure 4 Kaplan-Meier subgroup analysis of OS and RFS in HCC patients with IVCTT. (A) The OS for HCC patients after LR or IMRT (44 vs. 62 patients) before PSM ( $\mathrm{P}=0.986$ ); (B) the RFS for HCC patients after LR or IMRT (44 vs. 62 patients) before $\mathrm{PSM}(\mathrm{P}=0.557)$; (C) the OS for HCC patients after LR or RT (24 vs. 24 patients) after PSM ( $\mathrm{P}=0.821)$; (D) the RFS for HCC patients after LR or IMRT (24 vs. 24 patients) after PSM (4D) ( $\mathrm{P}=0.657)$. OS, overall survival; RFS, recurrence-free survival; HCC, hepatocellular carcinoma; IVCTT, inferior vena cava tumor thrombus; IMRT, intensity-modulated radiation therapy; LR, liver resection; PSM, propensity score matching.

\section{Discussion}

Macrovascular invasion has been well recognized as one of the most important poor prognostic factors of long-term survival in HCC patients $(24,25)$. The prognosis for HCC patients with HVTT is extremely poor $(3,26)$. Paucity of data on management of HCC patients with HVTT has made treatment decisions difficult, and the optimal choice of treatment for this subgroup of patients remains controversial. Recently, a Japanese nationwide survey demonstrated that LR produced reasonably good prognosis in selected HCC patients with HVTT, especially in those without PVTT (9). This large sample size study showed superiority of LR over non-LR treatments.
Recent developments in radiation techniques have enabled safe delivery of dose-escalated conformal radiotherapy to a wide spectrum of patients with inoperable HCC and liver metastases $(27,28)$. Promising clinical data on radiotherapy for unresectable HCC suggested that HCC is radiosensitive, with sustained local control rates ranging from $71 \%$ to $100 \%(29,30)$. Recently, RT was shown to be effective in treating unresectable HCC with PVTT. Lin et al. reported that stereotactic or three-dimensional conformal RT (3D-CRT) could result in recanalization of PVTT in unresectable HCC and that the responders showed significantly better 1 - and 2-year OS rates than non-responders (31).

The optimal treatment for HCC patients with HVTT 
remains highly debatable. Our study demonstrated that patients with HVTT undergoing LR had significantly better long-term OS than those undergoing IMRT, indicating that in selected patients LR was the better treatment for these patients. Moreover, Nakano et al. demonstrated that surgery provided better long-term OS and diseasefree survival in patients with small HCC tumors (32). On subgroup analysis, LR was a better treatment than IMRT for HCC patients with pHVTT and nHVTT, but not for patients with IVCTT. In our study, 138 of 307 HVTT patients (45\%) also had PVTT. The coexistent rate of PVTT and HVTT in our study was higher than those reported by others $(3,18,29)$.

This coexistence had a poorer prognosis than HVTT alone. Recent studies showed epithelial-mesenchymal transition (EMT), the mechanism involved in invasion in a variety of cancers (33), to be involved in vascular invasion in HCC. This may explain why there was a high coexistent rate of PVTT and HVTT in our study. This high coexistent rate was also found to be a significant risk factor of OS and RFS on univariate and multivariate analyses before and after PSM. However, why PVTT more commonly occurs than HVTT is unclear and requires further research.

For patients with IVCTT, available medical evidence to guide treatment is scarce, probably because of its rarity and the technical difficulty in its treatment. Wang et al. conducted a retrospective study on 56 IVCTT patients and showed significantly better MST in the 25 patients treated with surgery (34). Another Japanese study with a large sample size which involved 245 IVCTT patients demonstrated survival benefit of surgical over nonsurgical treatment (9). These two studies demonstrated surgery, although technically challenging, could safely be performed in IVCTT patients and resulted in better long-term survival outcomes than non-surgical treatment. In our study, LR and IMRT resulted in similar long-term survival in IVCTT patients. Such a result is probably because of the better local control capacity on IVCTT by the improved technology of irradiation using IMRT. A study from Japan also demonstrated beneficial effects of another improved radiation technology of 3D-CRT on HCC with IVCTT $(35,36)$. A novel RT technology, particle radiotherapy, has emerged to be a potential treatment for HCC patients with stage IIIB IVCTT and stage IV disease (37,38).

This study had several limitations. First, the etiology of HCC in this large series coming from China is mainly hepatitis B virus infection. Thus, the results of this study may not be applicable to HCC with other etiologies.
Second, it was difficult to select HCC patients with only HVTT without coexisting PVTT due to the high incidence of PVTT and the low incidence of HVTT. Thus, there is a high percentage of patients with coexistence of HVTT and PVTT in our study. Third, this is a retrospective study with its inherent defects.

In conclusion, LR was associated with better survival outcomes in HCC patients with hepatic vein tumor thrombus (pHVTT and mHVTT) than IMRT. However, for HCC patients with IVCTT, LR showed no survival benefit compared to IMRT.

\section{Acknowledgments}

Funding: This work was supported by the Key Project of the Natural Science Foundation of China (No. 81730097), grants of the Science Fund for Creative Research Groups (No. 81521091), the National Key Basic Research Program "973 Project" (No. 2015CB554000), the National Natural Science Foundation of China (No. 81602523 and No. $81702335)$.

\section{Footnote}

Reporting Checklist: The authors have completed the STROBE reporting checklist. Available at https://hbsn. amegroups.com/article/view/10.21037/hbsn.2020.03.20/rc

Data Sharing Statement: Available at https://hbsn. amegroups.com/article/view/10.21037/hbsn.2020.03.20/dss

Conflicts of Interest: All authors have completed the ICMJE uniform disclosure form (available at https://hbsn.amegroups. com/article/view/10.21037/hbsn.2020.03.20/coif). The authors have no conflicts of interest to declare. Dr. WYL serves as the unpaid editorial board members of Hepatobiliary Surgery and Nutrition. The other authors have no conflicts of interest to declare.

Ethical Statement: The authors are accountable for all aspects of the work in ensuring that questions related to the accuracy or integrity of any part of the work are appropriately investigated and resolved. The study was conducted in accordance with the Declaration of Helsinki (as revised in 2013). The study was approved by the Research Ethics Committee of the Eastern Hepatobiliary Surgery Hospital (Permit Number: HBHKY-2019-001-017). Informed consent was obtained from all the patients prior 
to treatment.

Open Access Statement: This is an Open Access article distributed in accordance with the Creative Commons Attribution-NonCommercial-NoDerivs 4.0 International License (CC BY-NC-ND 4.0), which permits the noncommercial replication and distribution of the article with the strict proviso that no changes or edits are made and the original work is properly cited (including links to both the formal publication through the relevant DOI and the license). See: https://creativecommons.org/licenses/by-nc-nd/4.0/.

\section{References}

1. Marrero JA, Kulik LM, Sirlin CB, et al. Diagnosis, staging, and management of hepatocellular carcinoma: 2018 practice guidance by the American Association for the Study of Liver Diseases. Hepatology 2018;68:723-50.

2. Kim HC, Lee JH, Chung JW, et al. Transarterial chemoembolization with additional cisplatin infusion for hepatocellular carcinoma invading the hepatic vein. J Vasc Interv Radiol 2013;24:274-83.

3. Kokudo T, Hasegawa K, Yamamoto S, et al. Surgical treatment of hepatocellular carcinoma associated with hepatic vein tumor thrombosis. J Hepatol 2014;61:583-8.

4. Le Treut YP, Hardwigsen J, Ananian P, et al. Resection of hepatocellular carcinoma with tumor thrombus in the major vasculature. A European case-control series. J Gastrointest Surg 2006;10:855-62.

5. Kodama K, Kawaoka T, Aikata H, et al. Comparison of outcome of hepatic arterial infusion chemotherapy combined with radiotherapy and sorafenib for advanced hepatocellular carcinoma patients with major portal vein tumor thrombosis. Oncology 2018;94:215-22.

6. Tsuzuki T, Ogata Y, Iida S, et al. Hepatic resection in 125 patients. Arch Surg 1984;119:1025-32.

7. Cheng AL, Kang YK, Chen Z, et al. Efficacy and safety of sorafenib in patients in the Asia-Pacific region with advanced hepatocellular carcinoma: a phase III randomised, double-blind, placebo-controlled trial. Lancet Oncol 2009;10:25-34.

8. Llovet JM, Ricci S, Mazzaferro V, et al. Sorafenib in advanced hepatocellular carcinoma. N Engl J Med 2008;359:378-90.

9. Kokudo T, Hasegawa K, Matsuyama Y, et al. Liver resection for hepatocellular carcinoma associated with hepatic vein invasion: a Japanese nationwide survey. Hepatology 2017;66:510-7.
10. Chen ZH, Zhang XP, Wang K, et al. Liver resection versus transcatheter arterial chemoembolization for the treatment of patients with hepatocellular carcinoma and hepatic vein or inferior vena cava tumor thrombus: a propensity score matching analysis. Hepatol Res 2019;49:441-52.

11. Zhang XP, Liu YC, Chen ZH, et al. Postoperative adjuvant transarterial chemoembolization improves outcomes of hepatocellular carcinoma associated with hepatic vein invasion: a propensity score matching analysis. Ann Surg Oncol 2019;26:1465-73.

12. Park W, Lim DH, Paik SW, et al. Local radiotherapy for patients with unresectable hepatocellular carcinoma. Int J Radiat Oncol Biol Phys 2005;61:1143-50.

13. Mornex F, Girard N, Beziat C, et al. Feasibility and efficacy of high-dose three-dimensional-conformal radiotherapy in cirrhotic patients with small-size hepatocellular carcinoma non-eligible for curative therapies--mature results of the French Phase II RTF-1 trial. Int J Radiat Oncol Biol Phys 2006;66:1152-8.

14. Seong J, Lee IJ, Shim SJ, et al. A multicenter retrospective cohort study of practice patterns and clinical outcome on radiotherapy for hepatocellular carcinoma in Korea. Liver Int 2009;29:147-52.

15. Xi M, Zhang L, Zhao L, et al. Effectiveness of stereotactic body radiotherapy for hepatocellular carcinoma with portal vein and/or inferior vena cava tumor thrombosis. PLoS One 2013;8:e63864.

16. Murakami E, Aikata H, Miyaki D, et al. Hepatic arterial infusion chemotherapy using 5-fluorouracil and systemic interferon- $\alpha$ for advanced hepatocellular carcinoma in combination with or without three-dimensional conformal radiotherapy to venous tumor thrombosis in hepatic vein or inferior vena cava. Hepatol Res 2012;42:442-53.

17. Tanaka Y, Nakazawa T, Komori S, et al. Radiotherapy for patients with unresectable advanced hepatocellular carcinoma with invasion to intrahepatic large vessels: efficacy and outcomes. J Gastroenterol Hepatol 2014;29:352-7.

18. Nakazawa T, Adachi S, Kitano M, et al. Potential prognostic benefits of radiotherapy as an initial treatment for patients with unresectable advanced hepatocellular carcinoma with invasion to intrahepatic large vessels. Oncology 2007;73:90-7.

19. Minagawa M, Makuuchi M. Treatment of hepatocellular carcinoma accompanied by portal vein tumor thrombus. World J Gastroenterol 2006;12:7561-7.

20. Shi J, Lai EC, Li N, et al. A new classification for hepatocellular carcinoma with portal vein tumor thrombus. 
J Hepatobiliary Pancreat Sci. 2011;18:74-80.

21. Wang K, Guo WX, Chen MS, et al. Multimodality treatment for hepatocellular carcinoma with portal vein tumor thrombus: a large-scale, multicenter, propensity mathching score analysis. Medicine (Baltimore) 2016;95:e3015.

22. Shi J, Lai EC, Li N, et al. Surgical treatment of hepatocellular carcinoma with portal vein tumor thrombus. Ann Surg Oncol 2010;17:2073-80.

23. Mizuno S, Kato H, Azumi Y, et al. Total vascular hepatic exclusion for tumor resection: a new approach to the intrathoracic inferior vena cava through the abdominal cavity by cutting the diaphragm vertically without cutting the pericardium. J Hepatobiliary Pancreat Sci 2010;17:197-202.

24. Pawlik TM, Poon RT, Abdalla EK, et al. Hepatectomy for hepatocellular carcinoma with major portal or hepatic vein invasion: results of a multicenter study. Surgery 2005;137:403-10.

25. Truty MJ, Vauthey JN. Surgical resection of high-risk hepatocellular carcinoma: patient selection, preoperative considerations, and operative technique. Ann Surg Oncol 2010;17:1219-25.

26. Florman S, Weaver M, Primeaux P, et al. Aggressive resection of hepatocellular carcinoma with right atrial involvement. Am Surg 2009;75:1104-8.

27. Ben-Josef E, Normolle D, Ensminger WD, et al. Phase II trial of high-dose conformal radiation therapy with concurrent hepatic artery floxuridine for unresectable intrahepatic malignancies. J Clin Oncol 2005;23:8739-47.

28. Lim DH, Lee H, Park HC, et al. The efficacy of high-dose 3-dimensional conformal radiation therapy in patients with small hepatocellular carcinoma not eligible for other local modalities. Am J Clin Oncol 2013;36:162-6.

29. Krishnan S, Dawson LA, Seong J, et al. Radiotherapy for hepatocellular carcinoma: an overview. Ann Surg Oncol 2008; 15:1015-24.

30. Hawkins MA, Dawson LA. Radiation therapy for

Cite this article as: Chen $\mathrm{ZH}$, Zhang XP, Feng S, Feng JK, Chai ZT, Guo WX, Shi J, Lau WY, Meng Y, Cheng SQ. Liver resection versus intensity-modulated radiation therapy for treatment of hepatocellular carcinoma with hepatic vein tumor thrombus: a propensity score matching analysis. HepatoBiliary Surg Nutr 2021;10(5):646-660. doi: 10.21037/hbsn.2020.03.20 hepatocellular carcinoma: from palliation to cure. Cancer 2006;106:1653-63.

31. Lin CS, Jen YM, Chiu SY, et al. Treatment of portal vein tumor thrombosis of hepatoma patients with either stereotactic radiotherapy or three-dimensional conformal radiotherapy. Jpn J Clin Oncol 2006;36:212-7.

32. Nakano R, Ohira M, Kobayashi T, et al. Hepatectomy versus stereotactic body radiotherapy for primary early hepatocellular carcinoma: a propensity-matched analysis in a single institution. Surgery 2018;164:219-26.

33. Wen W, Ding J, Sun W, et al. Cyclin G1-mediated epithelial-mesenchymal transition via phosphoinositide 3-kinase/Akt signaling facilitates liver cancer progression. Hepatology 2012;55:1787-98.

34. Wang Y, Yuan L, Ge RL, et al. Survival benefit of surgical treatment for hepatocellular carcinoma with inferior vena cava/right atrium tumor thrombus: results of a retrospective cohort study. Ann Surg Oncol 2013;20:914-22.

35. Zeng ZC, Fan J, Tang ZY, et al. A comparison of treatment combinations with and without radiotherapy for hepatocellular carcinoma with portal vein and/or inferior vena cava tumor thrombus. Int J Radiat Oncol Biol Phys 2005;61:432-43.

36. Igaki H, Nakagawa K, Shiraishi K, et al. Threedimensional conformal radiotherapy for hepatocellular carcinoma with inferior vena cava invasion. Jpn J Clin Oncol 2008;38:438-44.

37. Komatsu S, Fukumoto T, Demizu Y, et al. The effectiveness of particle radiotherapy for hepatocellular carcinoma associated with inferior vena cava tumor thrombus. J Gastroenterol 2011;46:913-20.

38. Komatsu S, Kido M, Asari S, et al. Particle radiotherapy, a novel external radiation therapy, versus liver resection for hepatocellular carcinoma accompanied with inferior vena cava tumor thrombus: a matched-pair analysis. Surgery 2017;162:1241-9. 
Supplementary

Table S1 Univariate and multivariable analysis for RFS in HCC patients with HVTT before PSM (n=307)

\begin{tabular}{|c|c|c|c|c|c|c|}
\hline \multirow{2}{*}{ Clinical variables } & \multicolumn{3}{|c|}{ Univariate analysis } & \multicolumn{3}{|c|}{ Multivariate analysis } \\
\hline & $\beta$ & $\mathrm{HR}(95 \% \mathrm{Cl})$ & $P$ & $\beta$ & $\mathrm{HR}(95 \% \mathrm{Cl})$ & $P$ \\
\hline Age, year & 0.000 & $1.000(0.990-1.011)$ & 0.915 & & & \\
\hline Sex, female vs. male & -0.180 & $0.835(0.597-1.167)$ & 0.292 & & & \\
\hline HBsAg, yes vs. no & 0.064 & $1.066(0.827-1.374)$ & 0.617 & & & \\
\hline Anti-viral treatment, yes vs. no & -0.094 & $0.910(0.558-1.480)$ & 0.706 & & & \\
\hline Satellite nodules, yes vs. no & 0.353 & $1.424(0.976-2.077)$ & 0.066 & & & \\
\hline \multicolumn{7}{|l|}{ Tumor capsulation } \\
\hline Incomplete vs. no & 0.266 & $1.305(0.866-1.966)$ & 0.203 & & & \\
\hline Complete vs. no & 0.040 & $1.041(0.720-1.506)$ & 0.828 & & & \\
\hline Lymph node invasion, yes vs. no & 0.156 & $1.169(0.819-1.667)$ & 0.387 & & & \\
\hline Ascites, yes vs. no & 0.449 & $1.566(1.110-2.210)$ & 0.010 & & & \\
\hline Multiple tumor, yes vs. no & -0.050 & $0.940(0.727-1.229)$ & 0.675 & & & \\
\hline \multicolumn{7}{|l|}{ AFP, ng/mL } \\
\hline $400-1,000$ vs. $<400$ & 0.104 & $1.109(0.711-1.732)$ & 0.648 & & & \\
\hline$\geq 1,000$ vs. $<400$ & 0.222 & $1.248(0.976-1.596)$ & 0.077 & & & \\
\hline Child-Pugh grade, A vs. B & 0.401 & $1.493(0.834-2.673)$ & 0.176 & & & \\
\hline Tumor diameter, cm & 0.030 & $1.030(0.998-1.063)$ & 0.019 & 0.038 & $1.039(1.005-1.073)$ & 0.021 \\
\hline PT, s & 0.019 & $1.019(0.949-1.095)$ & 0.590 & & & \\
\hline$A L T, U / L$ & 0.000 & $0.998(0.996-0.999)$ & 0.022 & & & \\
\hline TP, g/L & 0.000 & $0.990(0.977-1.010)$ & 0.692 & & & \\
\hline ALB, $g / L$ & 0.000 & $0.993(0.968-1.019)$ & 0.641 & & & \\
\hline AST, U/L & 0.000 & $0.996(0.994-0.990)$ & 0.007 & -0.003 & $0.997(0.994-0.999)$ & 0.026 \\
\hline GGT, U/L & 0.000 & $0.999(0.998-1.000)$ & 0.261 & & & \\
\hline ALP, U/L & 0.000 & $0.900(0.998-1.001)$ & 0.578 & & & \\
\hline PLT, $10^{\circ} / \mathrm{L}$ & 0.000 & $1.000(0.999-1.002)$ & 0.278 & & & \\
\hline TBIL, $\mu \mathrm{mol} / \mathrm{L}$ & 0.000 & $0.996(0.991-1.001)$ & 0.131 & & & \\
\hline $\mathrm{DBIL}, \mu \mathrm{mol} / \mathrm{L}$ & -0.010 & $0.980(0.962-1.001)$ & 0.070 & & & \\
\hline \multicolumn{7}{|l|}{ Extent of HVTT } \\
\hline mHCTT vs. pHVTT & 0.325 & $1.385(1.037-1.849)$ & 0.027 & 0.287 & $1.332(0.980-1.810)$ & 0.066 \\
\hline IVCTT vs. pHVTT & 1.014 & $2.757(2.037-3.733)$ & $<0.001$ & 1.127 & $3.080(2.252-4.200)$ & $<0.001$ \\
\hline \multicolumn{7}{|l|}{ Extent of PVTT } \\
\hline Type I vs. no & 0.305 & 1.357 (1.023-1.79) & 0.033 & 0.487 & $1.627(1.209-2.189)$ & 0.001 \\
\hline Type II vs. no & 0.278 & $1.321(0.961-1.814)$ & 0.085 & 0.307 & $1.359(0.976-1.894)$ & 0.069 \\
\hline Treatment, LR vs. IMRT & -0.400 & $0.665(0.526-0.841)$ & $<0.001$ & -0.727 & $0.483(0.370-0.623)$ & $<0.001$ \\
\hline
\end{tabular}

RFS, recurrence-free survival; HCC, hepatocellular carcinoma; HVTT, hepatic vein tumor thrombus; PSM, propensity score matching; IMRT, intensity-modulated radiation therapy; LR, liver resection; HBsAg, hepatitis B surface antigen; AFP, $\alpha$-fetoprotein; PT, prothrombin time; ALT, alanine aminotransferase; TP, total protein; ALB, albumin; AST, aspartate aminotransferase; GGT, $\gamma$-glutamyltransferase; ALP, alkaline phosphatase; PLT, platelet; TBIL, total bilirubin; DBIL, direct bilirubin; pHVTT, peripheral type of HVTT; mHVTT, major type of HVTT; IVCTT, inferior vena cava tumor thrombus; PVTT, portal vein tumor thrombus. 
Table S2 Univariate and multivariable analysis for OS in HCC patients with HVTT after PSM (n=164)

\begin{tabular}{|c|c|c|c|c|c|c|}
\hline \multirow{2}{*}{ Variables } & \multicolumn{3}{|c|}{ Univariate analysis } & \multicolumn{3}{|c|}{ Multivariate analysis } \\
\hline & $\beta$ & $\mathrm{HR}(95 \% \mathrm{Cl})$ & $P$ & $\beta$ & $\mathrm{HR}(95 \% \mathrm{Cl})$ & $P$ \\
\hline Age, year & -0.006 & $0.994(0.980-1.008)$ & 0.441 & & & \\
\hline Sex, female vs. male & -0.052 & $0.949(0.580-1.552)$ & 0.836 & & & \\
\hline HBsAg, yes vs. no & -0.210 & $0.810(0.577-1.136)$ & 0.223 & & & \\
\hline Anti-viral treatment, yes vs. no & 0.211 & $1.234(0.859-1.773)$ & 0.254 & & & \\
\hline Satellite nodules, yes vs. no & -0.015 & $0.980(0.570-1.700)$ & 0.958 & & & \\
\hline \multicolumn{7}{|l|}{ Tumor capsulation } \\
\hline Incomplete vs. no & -0.052 & $0.948(0.517-1.741)$ & 0.866 & & & \\
\hline Complete vs. no & -0.180 & $0.835(0.489-1.424)$ & 0.509 & & & \\
\hline Lymph node invasion, yes vs. no & -0.005 & $0.994(0.615-1.608)$ & 0.983 & & & \\
\hline Ascites, yes vs. no & 0.461 & $1.586(1.050-2.395)$ & 0.028 & & & \\
\hline Multiple tumor, yes vs. no & -0.106 & $0.899(0.620-1.302)$ & 0.574 & & & \\
\hline \multicolumn{7}{|l|}{ AFP, ng/mL } \\
\hline $400-1,000$ vs. $<400$ & 0.009 & $1.009(0.553-1.842)$ & 0.976 & & & \\
\hline$\geq 1,000$ vs. $<400$ & -0.009 & $0.991(0.712-1.380)$ & 0.959 & & & \\
\hline Child-Pugh grade, A vs. B & 1.162 & $3.000(0.995-10.25)$ & 0.051 & & & \\
\hline Tumor diameter, $\mathrm{cm}$ & 0.005 & $1.000(0.961-1.050)$ & 0.819 & & & \\
\hline PT, s & -0.003 & $0.997(0.875-1.136)$ & 0.966 & & & \\
\hline ALT, U/L & 0.000 & $0.999(0.996-1.002)$ & 0.766 & & & \\
\hline TP, g/L & -0.003 & $0.996(0.970-1.020)$ & 0.810 & & & \\
\hline ALB, g/L & 0.006 & $1.005(0.968-1.043)$ & 0.772 & & & \\
\hline AST, U/L & -0.003 & $0.996(0.992-1.000)$ & 0.126 & & & \\
\hline GGT, U/L & -0.001 & $0.999(0.998-1.000)$ & 0.273 & & & \\
\hline ALP, U/L & 0.000 & $0.999(0.997-1.001)$ & 0.629 & & & \\
\hline $\mathrm{PLT}, 10^{9} / \mathrm{L}$ & 0.000 & $1.000(0.998-1.002)$ & 0.929 & & & \\
\hline TBIL, $\mu \mathrm{mol} / \mathrm{L}$ & 0.003 & $1.002(0.997-1.008)$ & 0.319 & & & \\
\hline DBIL, $\mu \mathrm{mol} / \mathrm{L}$ & 0.008 & $1.007(0.975-1.040)$ & 0.637 & & & \\
\hline \multicolumn{7}{|l|}{ Extent of HVTT } \\
\hline mHCTT vs. pHVTT & 0.033 & $1.033(0.693-1.542)$ & 0.870 & 0.098 & $1.103(0.735-1.653)$ & 0.635 \\
\hline IVCTT vs. pHVTT & 1.073 & $2.922(1.959-4.358)$ & $<0.001$ & 1.407 & $4.080(2.650-6.294)$ & $<0.001$ \\
\hline \multicolumn{7}{|l|}{ Extent of PVTT } \\
\hline Type I vs. no & 0.050 & $1.051(0.728-1.517)$ & 0.789 & & & \\
\hline Type II vs. no & 0.392 & $1.480(0.954-2.295)$ & 0.080 & & & \\
\hline Treatment, LR vs. IMRT & -0.918 & $0.399(0.288-0.551)$ & $<0.001$ & -1.139 & $0.320(0.227-0.450)$ & $<0.001$ \\
\hline
\end{tabular}

OS, overall survival; HCC, hepatocellular carcinoma; HVTT, hepatic vein tumor thrombus; PSM, propensity score matching; IMRT, intensity-modulated radiation therapy; LR, liver resection; HBsAg, hepatitis B surface antigen; AFP, $\alpha$-fetoprotein; PT, prothrombin time; ALT, alanine aminotransferase; TP, total protein; ALB, albumin; AST, aspartate aminotransferase; GGT, $\gamma$-glutamyltransferase; ALP, alkaline phosphatase; PLT, platelet; TBIL, total bilirubin; DBIL, direct bilirubin; PHVTT, peripheral type of HVTT; mHVTT, major type of HVTT; IVCTT, inferior vena cava tumor thrombus; PVTT, portal vein tumor thrombus. 
Table S3 Univariate and multivariable analysis for RFS in HCC patients with HVTT after PSM (n=164)

\begin{tabular}{|c|c|c|c|c|c|c|}
\hline \multirow{2}{*}{ Clinical variables } & \multicolumn{3}{|c|}{ Univariate analysis } & \multicolumn{3}{|c|}{ Multivariate analysis } \\
\hline & $\beta$ & $\mathrm{HR}(95 \% \mathrm{Cl})$ & $P$ & $\beta$ & $\mathrm{HR}(95 \% \mathrm{Cl})$ & $P$ \\
\hline Age, year & 0.000 & $0.993(0.980-1.007)$ & 0.380 & & & \\
\hline Sex, female vs. male & -0.100 & $0.901(0.549-1.477)$ & 0.680 & & & \\
\hline HBsAg, yes vs. no & -0.190 & $0.823(0.586-1.156)$ & 0.261 & & & \\
\hline Anti-viral treatment, yes vs. no & 0.175 & $1.190(0.731-1.937)$ & 0.482 & & & \\
\hline Satellite nodules, yes vs. no & 0.035 & $1.035(0.598-1.791)$ & 0.900 & & & \\
\hline \multicolumn{7}{|l|}{ Tumor capsulation } \\
\hline Incomplete vs. no & 0.009 & $1.009(0.544-1.871)$ & 0.975 & & & \\
\hline Complete vs. no & -0.090 & $0.900(0.529-1.547)$ & 0.715 & & & \\
\hline Lymph node invasion, yes vs. no & 0.080 & $1.091(0.673-1.768)$ & 0.721 & & & \\
\hline Ascites, yes vs. no & 0.587 & $1.800(1.180-2.744)$ & 0.000 & 0.478 & $1.613(1.049-2.480)$ & 0.029 \\
\hline Multiple tumor, yes vs. no & -0.060 & $0.939(0.648-1.359)$ & 0.739 & & & \\
\hline \multicolumn{7}{|l|}{ AFP, ng/mL } \\
\hline $400-1,000$ vs. $<400$ & 0.055 & $1.057(0.598-1.866)$ & 0.850 & & & \\
\hline$\geq 1,000$ vs. $<400$ & -0.092 & $1.096(0.784-1.532)$ & 0.591 & & & \\
\hline Child-Pugh grade, A vs. B & 0.810 & $2.255(0.709-7.172)$ & 0.168 & & & \\
\hline Tumor diameter, cm & 0.000 & $1.009(0.966-1.054)$ & 0.669 & & & \\
\hline PT, s & 0.000 & $0.974(0.850-1.112)$ & 0.703 & & & \\
\hline$A L T, U / L$ & 0.000 & $0.998(0.990-1.001)$ & 0.431 & & & \\
\hline TP, g/L & 0.000 & $0.993(0.967-1.019)$ & 0.627 & & & \\
\hline ALB, $g / L$ & 0.011 & $1.011(0.976-1.047)$ & 0.530 & & & \\
\hline AST, U/L & 0.000 & $0.996(0.991-1.000)$ & 0.074 & & & \\
\hline GGT, U/L & 0.000 & $0.999(0.998-1.00)$ & 0.101 & & & \\
\hline ALP, U/L & 0.000 & $0.999(0.997-1.001)$ & 0.656 & & & \\
\hline PLT, $10^{\circ} / \mathrm{L}$ & 0.000 & $1.000(0.998-1.002)$ & 0.800 & & & \\
\hline TBIL, $\mu \mathrm{mol} / \mathrm{L}$ & 0.000 & $1.000(0.990-1.010)$ & 0.929 & & & \\
\hline DBIL, $\mu \mathrm{mol} / \mathrm{L}$ & 0.000 & $1.000(0.966-1.035)$ & 0.981 & & & \\
\hline \multicolumn{7}{|l|}{ Extent of HVTT } \\
\hline mHCTT vs. pHVTT & 0.270 & $1.310(0.883-1.944)$ & 0.178 & 0.371 & $1.449(0.969-2.160)$ & 0.070 \\
\hline IVCTT vs. pHVTT & 1.083 & $2.950(1.940-4.501)$ & $<0.001$ & 1.269 & $3.558(2.283-5.545)$ & $<0.001$ \\
\hline \multicolumn{7}{|l|}{ Extent of PVTT } \\
\hline Type I vs. no & -0.010 & $0.988(0.689-1.418)$ & 0.951 & & & \\
\hline Type II vs. no & 0.135 & $1.145(0.735-1.784)$ & 0.548 & & & \\
\hline Treatment, LR vs. IMRT & -0.930 & $0.391(0.282-0.541)$ & $<0.001$ & -1.119 & $0.326(0.230-0.458)$ & $<0.001$ \\
\hline
\end{tabular}

RFS, recurrence-free survival; HCC, hepatocellular carcinoma; HVTT, hepatic vein tumor thrombus; PSM, propensity score matching; IMRT, intensity-modulated radiation therapy; LR, liver resection; HBsAg, hepatitis B surface antigen; AFP, $\alpha$-fetoprotein; PT, prothrombin time; ALT, alanine aminotransferase; TP, total protein; ALB, albumin; AST, aspartate aminotransferase; GGT, $\gamma$-glutamyltransferase; ALP, alkaline phosphatase; PLT, platelet; TBIL, total bilirubin; DBIL, direct bilirubin; pHVTT, peripheral type of HVTT; mHVTT, major type of HVTT; IVCTT, inferior vena cava tumor thrombus; PVTT, portal vein tumor thrombus. 

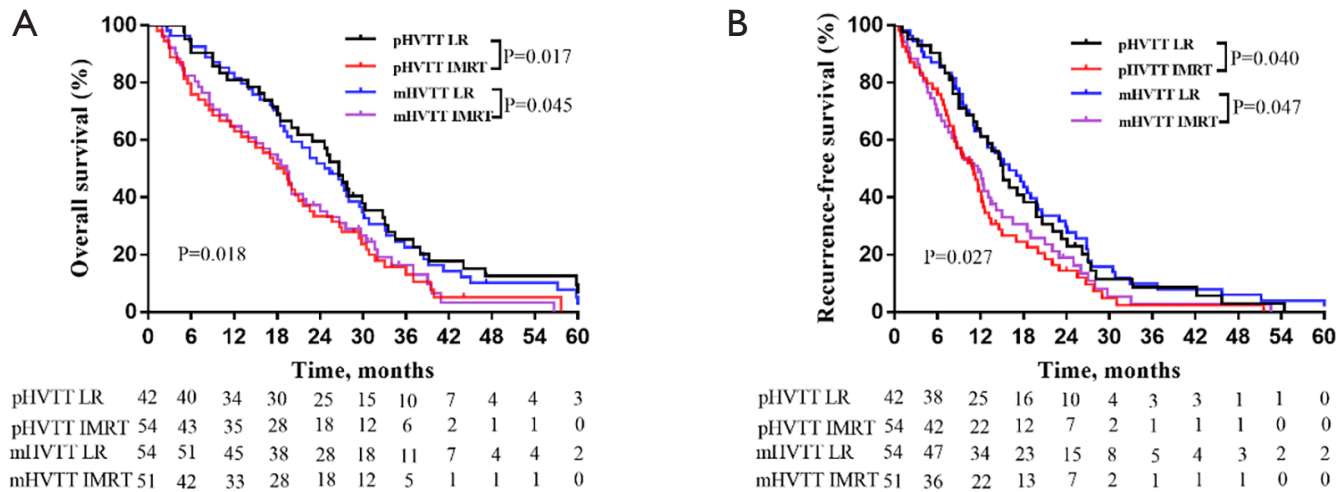

Figure S1 Kaplan-Meier analysis of OS and RFS of HCC patients with pHVTT and mHVTT of HVTT on subgroup analysis. (A) The OS for HCC patients after LR or IMRT (42 vs. 54 patients in pHVTT; 54 vs. 51 patients in mHVTT) after LR (P=0.018); (B) the RFS for HCC patients after LR or IMRT (42 vs. 54 patients in pHVTT; 54 vs. 51 patients in mHVTT) after LR (P=0.027). OS, overall survival; RFS, recurrence-free survival; HCC, hepatocellular carcinoma; HVTT, hepatic vein tumor thrombus; pHVTT, peripheral type of HVTT; mHVTT, major type of HVTT; IMRT, intensity-modulated radiation therapy; LR, liver resection.

A

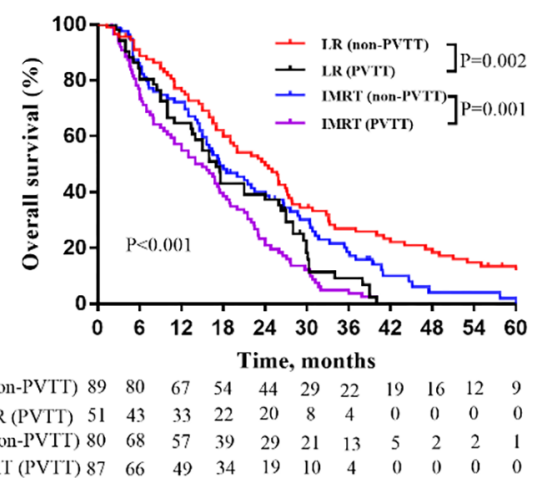

B

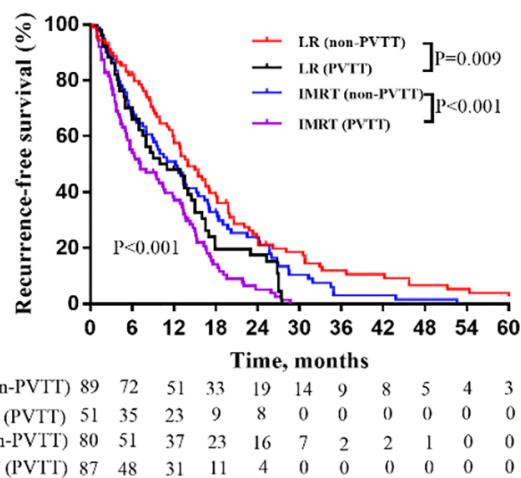

Figure S2 Kaplan-Meier analysis of OS and RFS in HCC patients with or without PVTT on subgroup analysis. (A) The OS for HCC patients with or without PVTT (87 vs. 80 patients after IMRT; 51 vs. 89 patients after LR) after LR (P<0.001); (B) the RFS for HCC patients with or without PVTT (87 vs. 80 patients after IMRT; 51 vs. 89 patients after LR) after LR (P<0.001). OS, overall survival; RFS, recurrence-free survival; HCC, hepatocellular carcinoma; PVTT, portal vein tumor thrombus; IMRT, intensity-modulated radiation therapy; LR, liver resection. 
Table S4 Adverse events of patients with HCC and HVTT who underwent IMRT

\begin{tabular}{llcl}
\hline & & No. of patients with CTCAE grade $(\mathrm{n})$ & 5 \\
\cline { 2 - 4 } Adverse event & 3 & 4 & - \\
\hline Fatigue & 3 & 2 & - \\
Anorexia & 2 & 2 & - \\
Nausea & 2 & 1 & - \\
ALT increase & 1 & - & - \\
Bilirubin increase & 1 & - & - \\
Gastroduodenitis & 1 & 1 & - \\
Gastric ulcer & 2 & 3 &
\end{tabular}

Adverse event was evaluated according to Common Terminology Criteria for Adverse Events (CTCAE) Version 4.0. HCC, hepatocellular carcinoma; HVTT, hepatic vein tumor thrombus; IMRT, intensity-modulated radiation therapy; ALT, alanine aminotransferase.

Table S5 Operative procedures and outcomes

\begin{tabular}{lc}
\hline Operative details & No. (\%) \\
\hline Major hepatectomy & $89(63.6)$ \\
Extent of resection & \\
R0 & $115(82.1)$ \\
R1 & $25(17.9)$ \\
Anatomical resection & $86(61.4)$ \\
Median hospital stay (days) & $19[14-24]$ \\
Clavien-dindo grade (total) & $45(32.1)$ \\
I & $22(15.7)$ \\
II & $18(12.9)$ \\
III & $3(2.1)$ \\
IV & $2(1.4)$ \\
V & 0 \\
\hline
\end{tabular}

Data are the median [IQR] or number (percentage) unless otherwise indicated. 